\title{
G protein-coupled estrogen receptor-1 agonist induces chemotherapeutic effect via ER stress signaling in gastric
}

\section{cancer}

\author{
Seon-Jin Lee ${ }^{1}$, Tae Woo Kim ${ }^{2}$, Gyeong Lim Park ${ }^{1}$, Yo Sep Hwang ${ }^{2}$, Hee Jun Cho ${ }^{2}$, Jong-Tae Kim ${ }^{2}$ E Hee Gu Lee ,* $^{2}$ \\ ${ }^{1}$ Environmental Disease Research Center, Korea Research Institute of Bioscience and Biotechnology, Daejeon 34141, ${ }^{2}$ Immunotherapy \\ Convergence Research Center, Korea Research Institute of Bioscience and Biotechnology, Daejeon 34141, Korea
}

\begin{abstract}
G protein-coupled estrogen receptor (GPER) is known to play an important role in hormone-associated cancers. G-1, a novel synthetic GPER agonist, has been reported to exhibit anti-carcinogenic properties. However, the chemotherapeutic mechanism of GPER is yet unclear. Here, we evaluated GPER expression in human gastric cancer tissues and cells. We found that G-1 treatment attenuates GPER expression in gastric cancer. GPER expression increased G-1-induced antitumor effects in mouse xenograft model. We analyzed the effects of knockdown/overexpression of GPER on G-1-induced cell death in cancer cells. Increased GPER expression in human gastric cancer cells increased $\mathrm{G}$-1-induced cell death via increased levels of cleaved caspase-3, -9 , and cleaved poly ADP-ribose polymerase. Interestingly, during G-1-induced cell death, GPER mRNA and protein expression was attenuated and associated with ER stress-induced expression of PERK, ATF-4, GRP-78, and CHOP. Furthermore, PERK-dependent induction of ER stress activation increased G-1-induced cell death, whereas PERK silencing decreased cell death and increased drug sensitivity. Taken together, the data suggest that the induction of ER stress via GPER expression may increase G-1-induced cell death in gastric cancer cells. These results may contribute to a new paradigm shift in gastric cancer therapy. [BMB Reports 2019; 52(11): 647-652]
\end{abstract}

\section{INTRODUCTION}

Gastric cancer is a common malignancy worldwide and one of the leading causes of tumor-related death. Several studies have been designed to develop therapeutic drugs for improved

${ }^{*}$ Corresponding author. Tel: +82-42-860-4182; Fax: +82-42-8604593; E-mail: hglee@kribb.re.kr

https://doi.org/10.5483/BMBRep.2019.52.11.007

Received 5 January 2019, Revised 9 February 2019, Accepted 8 April 2019

Keywords: Cell death, ER stress, G-1, Gastric cancer, GPER disease prognosis $(1,2)$ and numerous chemotherapeutic regimens have been clinically investigated. However, novel therapeutic agents that improve patient survival are urgently required. Clinical data showed that the incidence of gastric cancer is significantly lower in women than in men, which may be attributed to the presence of estrogens (3).

G protein-coupled estrogen receptor (GPER), a member of G protein-coupled receptors (GPCRs), has a diverse array of functions in many tissues, such as breast, and ovary $(4,5)$. The physiological role of GPER in regulating the functions of the endocrine and reproductive systems has also been evaluated $(4,5)$. Recent reports revealed that GPER contributes to pathophysiological responses, such as tumor proliferation, and migration, particularly during breast cancer development (6). On the other hand, many studies have demonstrated that activation of GPER, which is a specific agonist of G-1 (1-[4-(6-bromobenzo [1, 3] dioxol-5yl)-3a, 4, 5, 9b-tetrahydro$3 \mathrm{H}$-cyclopenta-[c]quinolin-8-yl]-ethanone) induces cell-cycle arrest and inhibition of ER $\alpha$-positive cell proliferation (4, 7). Therefore, further investigation is necessary to elucidate the activation of GPR30 and the effect of G-1 on cancer cells.

Recently, the GPER agonist role of G-1 has been evaluated in GPER-mediated estrogen regulation compared with those mediated by estrogen receptors such as $\mathrm{ER} \alpha$ and $\beta$ (8). G-1 has also been studied in numerous studies to demonstrate the role of GPER in immune system and cancer (9). The biochemical activities triggered by G-1-mediated GPER activation following cell proliferation (6) and/or cell death $(4,7)$, appear to be cell-type-specific and dependent on the pattern of ERs expression (4). The identification of the role of G-1 in the activity of ER $\alpha$ or 25 other important GPCRs (10) facilitated the study of GPER-mediated signal transduction and downregulation of G-1-induced urothelial cell proliferation (11) and attenuation of prostate cancer proliferation (7). These reports suggested that GPER may induce a functional interaction between tumors and tumor components (12). Herein, we investigated the mechanism of G-1-induced cell mortality in gastric cancer cells and described the GPER-mediated tumor suppressive effects. Our results suggest a potential therapeutic role in gastric cancer.

ISSN: 1976-670X (electronic edition)

Copyright (c) 2019 by the The Korean Society for Biochemistry and Molecular Biology

(c) This is an open-access article distributed under the terms of the Creative Commons Attribution Non-Commercial License (http://creativecommons.org/licenses/by-nc/4.0) which permits unrestricted non-commercial use, distribution, and reproduction in any medium, provided the original work is properly cited. 


\section{RESULTS}

\section{Stage-dependent decrease in GPER expression of gastric cancer and correlation with resistance to $\mathrm{G}$-1-induced cell death}

The pattern of GPER expression in differential tumor stages of gastric cancer was analyzed using immunohistochemical (IHC) staining of normal and cancerous gastric tissue array samples derived from 55 gastric cancer patients (Fig. 1A). GPER fluorescence intensity decreased about two-fold in tumor stages I and II (45\% and 30\%) compared with stages III and IV $(25 \%$ and $20 \%)$. We investigated GPER mRNA levels using qPCR analysis of normal and gastric tumor tissues (Fig. 1B). GPER mRNA levels were about five-fold lower in cancer than in normal tissues. We also analyzed the levels of GPER mRNA and protein in the following gastric cancer cell lines: AGS, SNU-216, NCI-N87, SNU-620, SNU-638, SNU-668, NUGC-3, and MKN-74 (Fig. 1C). GPER mRNA and protein levels were approximately 10 -fold higher in gastric cancer cells, AGS, and SNU-216 compared with NCl-N87, SNU-620, SNU-638, SNU-668, NUGC-3, and MKN-74. Next, we tested the mortality of gastric cancer cell lines in a dose-dependent response to GPER agonist (G-1) using WST-1 assay (Fig. 1D).

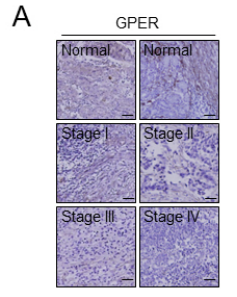

C

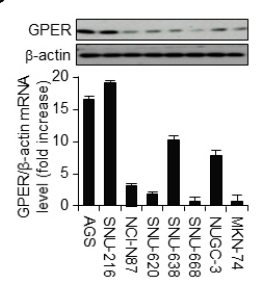

B

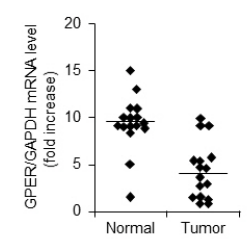

D

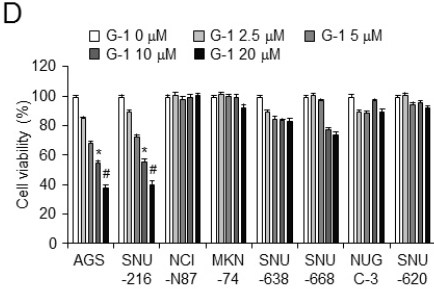

Fig. 1. GPER expression is downregulated in vivo and in vitro in late-stage gastric cancer. (A) Representative immunohistochemical images (left) and microarray-based quantitation (right) of GPER expression in normal $(\mathrm{n}=59)$ and gastric cancer tissues at indicated stages (stage I, $\mathrm{n}=8 ; \mathrm{II}, \mathrm{n}=14$; III, $\mathrm{n}=24$; and IV, $\mathrm{n}=3$ ); original magnification, 200X; scale bars, $50 \mu \mathrm{m}$; (B) GPER expression in 16 pairs of normal and cancer tissues from gastric cancer patients normalized to the GAPDH expression determined with qPCR; (C) Cell viability of gastric cancer cells treated with G-1 at the indicated doses measured by WST-1 assay. The viability of drug-treated cells was expressed relative to that of DMSO-treated control cells, whose viability was set at $100 \%$. (D) Western blotting (top) and quantitation of real-time RT-PCR (bottom) data of GPER expression in the indicated cell lines. ${ }^{*} P<0.05,{ }^{\#} P<0.01$ versus control.

\section{GPER agonist enhances G-1-induced tumor suppression in vivo and in vitro}

We tested whether these results suggesting that GPER expression level was a crucial factor in G-1-mediated cell death could be demonstrated in vivo. Mice were administered G-1 intraperitoneally (i.p.) to analyze its effects based on the GPER level using AGS, SNU-216, which contains high GPER-expressing cells, and NCl-N87, which contains few GPER-expressing cells. Effects of GPER expression and G-1 treatment on tumor volume are presented in Fig. 2A. G-1 treatment decreased the tumor volume comprising both AGS and SNU-216 cells. These results clearly suggest that GPER agonist has a strong potential to inhibit the tumor growth of gastric cancer cells. As high GPER expression appeared to correlate with chemotherapeutic effect of GPER agonist (G-1) in vivo, we further demonstrated the relationship between GPER expression and cell viability in G-1-treated cells via assays for Annexin $\mathrm{V}$ apoptosis, western blotting, and nuclear fragmentation (Fig. 2B-D). Following G-1 treatment, NCl-N87 cells showed few Annexin V-positive and DAPI-positive cells, whereas AGS cells contained an increase in Annexin V-positive cells (Fig. 2B). We also investigated whether the induction of apoptotic events attenuated the viability of G-1-sensitive cells AGS and SNU-216 following cleavage of
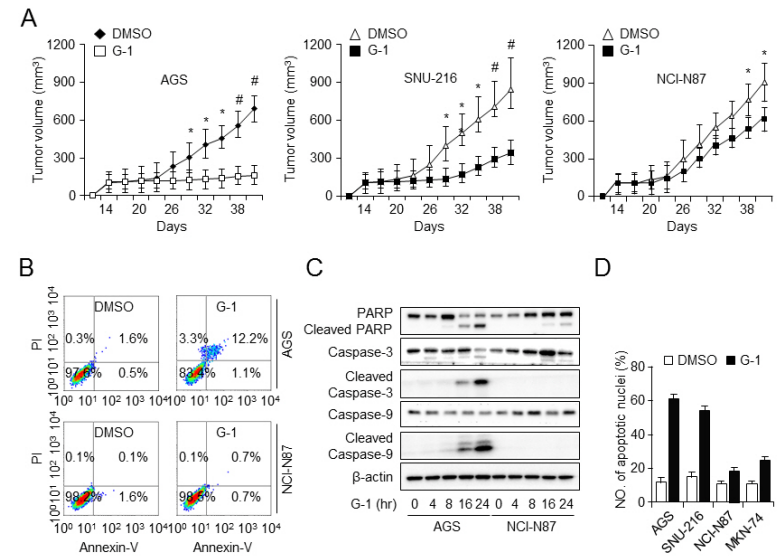

Fig. 2. GPER agonist increases G-1-induced cell death in xenograft tumor and gastric cancer cells. (A) Tumor volumes on the indicated days after injection of AGS (left graph), SNU-216 (middle graph) cells, and $\mathrm{NCl}-\mathrm{N} 87$ cells (right graph) in the presence or absence of G-1 treatment into mice. Values represent the mean \pm SD for each group $(\mathrm{n}=8)$ (B) FACS analysis of Annexin $\mathrm{V}$ expression in AGS (top) and NCl-N87 (bottom) cells treated with DMSO $(\mathrm{CTL})$ or $\mathrm{G}-1(2.5 \mu \mathrm{M})$. (C) Western blotting of cleaved caspase-3, caspase-9, and PARP in the indicated cell types treated with G-1 $(2.5 \mu \mathrm{M})$ for the indicated times. (D) AGS, SNU-216, NCl-N87, and MKN-74 cells were treated with or without G-1 treatment $(2.5 \mu \mathrm{M}, 8 \mathrm{~h})$. After G-1 treatment, nuclei were stained with DAPI and quantified graphically using a confocal microscope (Zeiss, LSM510). ${ }^{* P}<0.05,{ }^{\#} \mathrm{P}<0.01$ versus control. 
caspase-3, caspase-9, and PARP (Fig. 2C). Nuclear fragmentation assay revealed that AGS and SNU-216 cells with high GPER expression increased the intensity of DAPI staining to G-1 treatment when compared with $\mathrm{NCl}-\mathrm{N} 87$ and MKN-74 cells with a lower GPER expression (Fig. 2D).

\section{Upregulation of GPER increases GPER agonist-induced gastric cancer cell death}

To examine the association between GPER expression and G-1-induced cell death in gastric cancer, we measured GPER mRNA levels in the gastric cancer cell lines following G-1 treatment at multiple time points. Interestingly, AGS and SNU-216 cells showed a decreased GPER mRNA expression with G-1 treatment $(2.5 \mu \mathrm{M})$ than the other cells lines, $\mathrm{NCl}-\mathrm{N} 87$ and MKN-74 (Fig. 3A). We also investigated the GPER protein expression in a time- and dose-dependent manner with G-1 treatment. The results suggested that GPER protein expression was similar to the mRNA expression pattern in gastric cancer cells and the cytotoxicity of G-1 may be dependent upon the expression of GPER (Fig. 3B). We hypothesized that the GPER expression is an important factor in G-1-induced cell mortality. To demonstrate that the loss/gain of GPER function influences G-1-induced cell mortality, we induced small interfering RNA (siRNA)-mediated
A

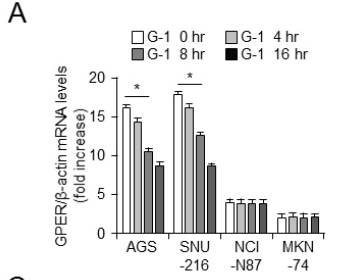

C
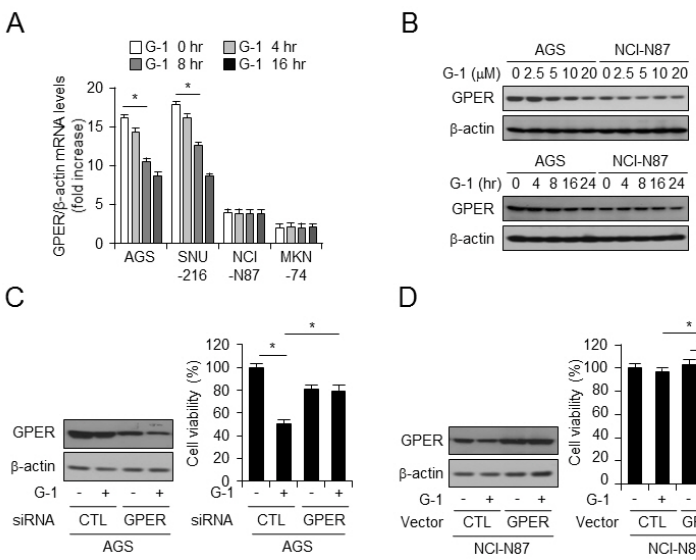

D

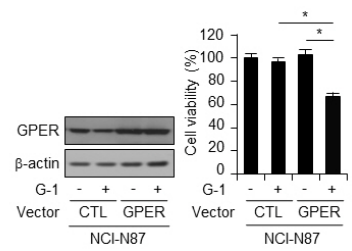

Fig. 3. GPER downregulation with G-1 treatment decreases therapeutic effects in gastric cancer cells. (A) GPER transcriptional levels at the indicated times in AGS, SNU-216, NCl-N87, and MKN-74 cells normalized to the $\beta$-actin expression level as determined with qPCR; (B) Western blotting analysis at the indicated doses (top) and time (bottom) in gastric cancer cell lines AGS and NCl-N87; (C) After transfection with control (CTL) or GPER siRNA in the presence and absence of G-1 $(2.5 \mu \mathrm{M})$ treatment, cell viability and expression of GPER in AGS cells were measured by WST-1 assay and western blotting; (D) Cell viability assay and western blot of GPER, cleaved caspase-3, and LC3B in NCl-N87 and AGS cells following transfection with GPER overexpression plasmid or mock vector in the presence and absence of G-1 $(2.5 \mu \mathrm{M})$. All data shown are the mean \pm SD of three independent experiments. ${ }^{*} P<0.05$ versus control.
GPER knockdown (siGPER) in high-GPER cells such as AGS, and transiently overexpressed GPER with the pcDNA3.1-GPER vector in the low-GPER cells such as NCI-N87. As shown in Fig. 3C, GPER deficiency in AGS cells showed a decrease in cell mortality and GPER expression after G-1 treatment. On the other hand, GPER overexpression in NCl-N87 cells resulted in a strong increase in cell mortality and GPER expression following G-1 treatment (Fig. 3D).

\section{GPER agonist increases G-1-induced cell death via ER stress in gastric cancer}

Next, we asked whether the GPER agonist stimulated G-1-induced ER stress signal transduction in gastric cancer cells. We analyzed the time-dependent transcription of ER stress proteins in G-1 treated gastric cancer cell lines and observed significant increases in GRP78, PERK, ATF4, and CHOP mRNA levels in G-1-sensitive AGS cells, which resulted in increased cell death compared with the G-1-resistant, $\mathrm{NCl}-\mathrm{N} 87$ cells (Fig. 4A). Furthermore, we wondered whether the GPER agonist activated ER stress pathway following G-1 treatment; however, AGS cells showed an increase in post-translational activation of ER stress markers including p-PERK, ATF4, GRP78, and CHOP upon G-1 treatment (Fig. $4 \mathrm{~B})$. To demonstrate that the loss/gain of PERK function influences G-1-mediated ER stress activation, we induced siRNA-mediated PERK knockdown (siPERK) in the high-GPER lines AGS and low-GPER lines NCl-N87 for the indicated time. As shown in Fig. 4C, siRNA-mediated PERK knockdown in

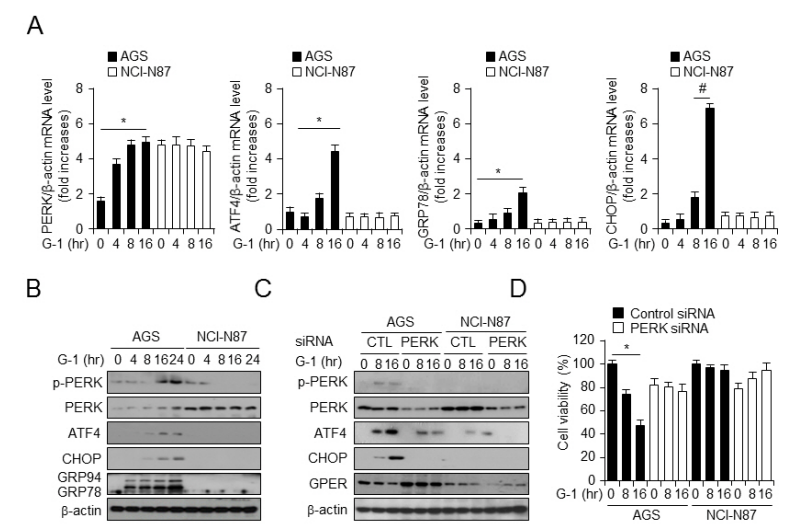

Fig. 4. GPER expression enhances G-1-induced inhibition of cell growth via ER stress in gastric cancer. (A) AGS and NCl-N87 cells treated with G-1 $(2.5 \mu \mathrm{M})$ at the indicated time, and samples of total lysates normalized with $\beta$-actin followed by qPCR to identify the level of ER stress-related genes PERK, ATF4, GRP78, CHOP; (B) Western blotting analysis of AGS and $\mathrm{NCl}-\mathrm{N} 87$ cells treated with G-1 using phosphor-PERK, PERK, ATF4, GRP78, and CHOP antibodies;. Western blotting (C) and WST-1 assay (D) after transfection of control or PERK-targeted siRNA into AGS and NCl-N87 cells treated with G-1. All data shown represent the mean \pm SD of three independent experiments. ${ }^{*} P<0.05,{ }^{\#} P<0.01$ versus control. 
AGS cells resulted in a decreased ER stress signal with decreased phosphorylation levels of PERK, and reduced ATF4 and CHOP expression while GPER expression was increased. These changes were not detected in the NCl-N87 cells. We also found that siRNA-mediated PERK knockdown resulted in marked increases in cell mortality and GPER expression following GPER agonist (G-1) treatment in AGS cells but not in $\mathrm{NCl}-\mathrm{N} 87$ cells (Fig. 4D).

\section{DISCUSSION}

Although estrogen-related signal transduction has been suggested to downregulate the tumor progression of gastric cancer cells, the functional role and mechanisms of GPER in the development of gastric cancer have not been demonstrated. Our results showed that the expression of GPER in tumor tissues was less than in the corresponding adjacent normal tissues. Activation of GPER by G-1 increases cell mortality, and the levels of cleaved caspase-3, -9 and cleaved PARP, and elevates ER stress in gastric cancer cells. Activation of GPER downregulates the growth of gastric cancer cells both in vitro and in vivo via ER stress signaling pathway.

Our studies showed that GPER might be an important target for gastric cancer treatment. Recent reports have indicated that the activation of GPER as G-1 suppresses the progression of multiple cancers, including prostate (7) and breast cancer (4). Our current study demonstrated that G-1 attenuated gastric cancer cell proliferation in vitro via ER stress-related apoptosis and also showed that G-1 treatment attenuated the growth of AGS and SNU-216 xenograft tumors in nude mice. Thus, GPER might modulate the protective role of estrogen-related signals in gastric cancer development and inhibit cancer progression. Other studies have reported that GPER expression initiated the proliferation of breast cancer cells (6), which may have contributed to the agonist specificities and differences in cell types and treatment conditions. As shown in Fig. 2, GPER activation may be related to stimulation of the intrinsic apoptotic mechanism. The intrinsic apoptotic mechanism activated in caspase-9, -3 , and PARP-1 levels in gastric cancer cells in response to GPER activation. Depletion of the GPER gene suggested a role of this receptor in the attenuation of cell viability.

We also found that PERK/ATF4/GRP78/CHOP proteins were enhanced in G-1-treated AGS and SNU-216 cells than in $\mathrm{NCl}-\mathrm{N} 87$ and MKN-74 cells. Moreover, our results also suggested that knockdown of each of the ER stress signal proteins such as PERK, with small interfering RNA, blocked the inhibition of tumor growth by G-1 in AGS. These findings suggested that GPER signaling-mediated ER stress in gastric cancer cells and G-1-induced enhancement of ER stress may promote gastric cancer cell death. CHOP is expressed at substantially low levels under normal conditions; however, it is highly upregulated during pathological stages and under prolonged ER stress, cell arrest and apoptosis activation (13).
The PERK/elF2 $\alpha /$ ATF4 signaling pathway plays an important role in activating CHOP transcription (14). When ER stress induces apoptosis, PERK activation attenuated translation and induction of ATF4, indicating CHOP activation (15). Our results demonstrated that G-1-induced ER stress increased the expression of CHOP by phosphorylating PERK/elF2 $\alpha /$ ATF4, as suggested by the increased levels of GRP78, p-elF2 $\alpha$, ATF4 and $\mathrm{CHOP}$ proteins in gastric cancer cells.

In conclusion, GPER may contribute to G-1-induced cell death and cancer growth inhibition in vitro and in vivo. Modulation of the GPER status in G-1-induced cell mortality is associated with potential therapeutic effects in gastric cancer. Co-treatment with GPER agonist and induction of ER stress using agents such as tunicamycin may result in further improvement of treatment efficacy depending on GPER agonists for the regulation of tumor growth. We provided evidence supporting a novel G-1 signal transduction mechanism mediated via activation of ER stress that is linked to the activation of PERK. We may develop chemotherapeutic strategies based on G-1-induced cell mortality in gastric cancer cells.

\section{MATERIALS AND METHODS}

\section{Cell culture}

The gastric cancer cell lines AGS, SNU-216, NCl-N87, SNU-620, SNU-638, SNU-668, NUGC-3, and MKN-74 were purchased from the Korean Cell Line Bank (Cancer Research Center, Seoul National University, Seoul, Korea) and grown in RPMI1640 medium (Gibco) supplemented with 5\% fetal bovine serum (Gibco) and $100 \mu \mathrm{g} / \mathrm{ml}$ antibiotics $(100 \mathrm{U} / \mathrm{ml}$ penicillin and $100 \mu \mathrm{g} / \mathrm{ml}$ streptomycin, Gibco). Cells $\left(1 \times 10^{5}\right.$ cells/well) were plated in 24-well cell culture plates and grown at $37^{\circ} \mathrm{C}$ in a humidified, $5 \% \mathrm{CO}_{2} /$ air atmosphere.

\section{Cell viability}

The WST-1 assay was performed based on the manufacturer's instructions (Roche, Mannheim) by adding $10 \mu \mathrm{l}$ of WST-1 reagent to each well of a 96-well plate $\left(1 \times 10^{3}\right.$ cell/well). After $1 \mathrm{~h}$ of incubation in a $\mathrm{CO}_{2}$ incubator, the conversion of WST-1 reagent into chromogenic formazan was monitored with a spectrophotometer.

\section{Transfection}

AGS and NCl-N87 cells $\left(1 \times 10^{5}\right.$ cell/well $)$ in a 24 -well plate were transfected with double-stranded siRNAs $(30 \mathrm{nmol} / \mathrm{ml}$ ) such as siGPER and siPERK (Bioneer) for $24 \mathrm{~h}$ via the Lipofectamine 2000 (Invitrogen) method based on the manufacturer's protocol, and recovered in RPMI1640 medium (Welgene) containing 10\% fetal bovine serum for $24 \mathrm{~h}$. After recovery, viable cells were calculated by the WST-1 Cell Proliferation Assay system. The pcDNA3.1 - GPER was purchased from Addgene (Watertown, MA). 


\section{Quantitative polymerase chain reaction}

Total RNA from gastric cells $\left(2 \times 10^{6}\right.$ cells/well $)$ in $100 \mathrm{~mm}$ cell culture dish and tissues (approximately 50-100 mg) was prepared using TRIzol following manufacturer's recommendations (Invitrogen, Carlsbad). Total RNA from cultured cells and tissues was prepared using an All Prep DNA/RNA Mini kit (Qiagen, Valencia) with elution of 100 and $30 \mu \mathrm{l}$, respectively. GPER, PERK, ATF4, GRP78, and CHOP expression levels were measured by qPCR analysis using CDNA synthesized from 5 $\mu \mathrm{g}$ of total RNA and a reverse transcription kit (Promega, Madison). One microliter of CDNA was used for the PCR, and duplicate reactions were performed for each sample using an ABI Power SYBR green PCR Master Mix (Applied Biosystems, Warrington) on an $A B I$ Step one plus instrument. We used oligonucleotide primer sequences specific for GPER (5'-AGT CGG ATG TGA GGT TCA G-3' and 5'-TCT GTG TGA GGA GTA CAA G-3'), PERK (5'-CGT GAT GGT TCA AGA CAT GC-3' and 5'-GTT CCC GAT GAA CTC AAG GA-3'), ATF4 (5'-AAG CCT AGG TCT CTT AGA TG-3' and 5'-TTC CAG GTC ATC TAT ACC CA-3'), GRP78 (5'-GTT CTT GCC GTT CAA GGT GG-3' and $5^{\prime}$-TGG TAC AGT AAC AAC TGC ATG-3'), and CHOP (5'-ATG AGG ACC TGC AAG AGG TCC-3' and 5'-TCC TCC TCA GTC AGC CAA GC-3'). The RNA quantity was normalized to $\beta$-actin content, and gene expression was quantified according to the $2^{-\Delta \mathrm{Ct}}$ method.

\section{Western blotting}

Cancer cell lines were solubilized in radio-immunoprecipitation assay (RIPA) lysis buffer (50 mML Tris- $\mathrm{HCl}(\mathrm{pH} 7.4)$, $150 \mathrm{mM} / \mathrm{L} \mathrm{NaCl}, 1 \% \mathrm{NP} 40,0.25 \%$ sodium deoxycholate, 1 $\mathrm{mM} / \mathrm{L}$ phenylmethylsulfonylfluoride (PMSF), $1 \mathrm{mM} / \mathrm{L}$ sodium orthovanadate, and $1 \mathrm{X}$ sigma protease inhibitor cocktail. The protein was measured using a standard bicinchoninic acid assay. Equal amounts of protein (20 to $50 \mu \mathrm{g}$ ) were size-fractionated by $\sim 10-15 \%$ SDS-PAGE and then transferred onto PVDF membrane. Membranes were blocked by incubation for $1 \mathrm{~h}$ with $5 \%$ skim milk/PBS-T buffer (PBS with $5 \%$ powdered milk and $1 \%$ Triton X-100), and incubated overnight at $4^{\circ} \mathrm{C}$ with primary antibodies diluted in $1 \mathrm{X}$ PBST buffer. The membranes were washed 3 times with PBST. Secondary antibodies were diluted in PBST and added for 40 min at room temperature. The following secondary antibodies were used: anti-rabbit IgG HRP-linked antibody and anti-mouse IgG HRP-linked antibody. The membranes were washed 6 times with PBST for $1 \mathrm{~h}$. The blots were visualized by chemiluminescence.

\section{Immunohistochemistry}

Tissue microarray slides for human gastric normal and tumor were purchased from SuperBioChips (SuperBioChips Laboratories, Seoul). Tissue sections were deparaffinized by xylene and blocked by normal serum. Slides were blocked with an anti-GPER (Santa Cruz Biotechnology, Inc.) primary antibody, diluted in CAS blocking solution (Invitrogen) overnight followed by overnight incubation in biotinylated secondary antibody. Tissue slides were washed and developed using the DAB substrate kit (DAB substrate kit, vector laboratories) at room temperature, and a mounting medium was used following the manufacturer's recommendations (VECTASTAIN ABC KIT, Vector laboratories).

\section{Flow cytometry and DAPI staining}

Cells were stained as described in the FITC Annexin V Apoptosis Detection Kit (BD Pharmingen, San Diego) and counted by flow cytometry (BD FACS Calibur) to detect apoptosis. FITC Annexin $\mathrm{V}$ Apoptosis Detection was performed following the manufacturer's instructions (BD Pharmingen).

For DAPI staining, changes in nuclear morphology of the gastric cancer cells $\left(1 \times 10^{5} /\right.$ well $)$ plated on a 6 -well plate undergoing apoptosis were detected by staining with DAPI (Sigma). After experiment, cells were washed twice with PBS and fixed with $4 \%$ paraformaldehyde for $30 \mathrm{~min}$. Following PBS washes, cells were incubated in a DAPI solution for 30 min in the dark. Cells were washed with PBS and subjected to fluorescence microscopy.

\section{Xenograft assay}

Female 6- to 8-week-old female athymic nu/nu nude mice were purchased from Central Lab, Animal Inc. (Seoul) and used for in vivo experiments. The experimental protocol was reviewed and approved by the Institutional Animal Care and Use Committee of the Korea Research Institute of Bioscience and Biotechnology (KRIBB). AGS and NCl-N87 cells were used in a xenograft assay. The mice were divided into DMSO and G-1 (1 mg/kg) treated groups (8 mice per each group) and administered G-1 intraperitoneally (i.p.) every other day. In the xenograft assay, $3 \times 10^{6}$ cells were collected by centrifugation and resuspended in $0.1 \mathrm{ml}$ of PBS, and injected subcutaneously (s.c.) into right dorsal flank of nude mice (eight mice per cell line) using 25-gauge needles. When tumor volumes showed approximately $200 \mathrm{~mm}^{3}$, treatment with G-1 was started and monitored twice weekly. Tumor volumes of mice were measured with calipers and calculated using the following formula: $\left(A \times B^{2}\right) / 2$, where $A$ is the largest and $B$ is the smallest diameter.

\section{Statistical analysis}

All results were confirmed in at least three independent experiments. Data represent a single experiment. All quantitative data are presented as mean \pm standard deviation (SD). In vivo data are expressed as mean \pm standard error of the mean (SEM). Statistical analysis was performed using SAS 9.2 software (SAS Institute, Cary). Student's t-tests were used for comparison of means of quantitative data between groups and $\mathrm{P}<0.05$ was considered statistically significant. 


\section{ACKNOWLEDGEMENTS}

This research was funded by NRF (National Research Foundation of South Korea)-2017R1A2B2005629 and 2017R 1A2B4006726 and supported by a grant obtained from KRIBB Research Initiative Program.

\section{CONFLICTS OF INTEREST}

The authors have no conflicting interests.

\section{REFERENCES}

1. Park KS, Hong YS, Choi JY et al (2018) HSP90 inhibitor, AUY922, debilitates intrinsic and acquired lapatinibresistant HER2-positive gastric cancer cells. BMB Rep 51, 660-665

2. Seo JS, Kee HJ, Choi HJ et al (2018) Inhibition of Wntless/ GPR177 suppresses gastric tumorigenesis. BMB Rep 51, 255-260

3. Simon MS, Chlebowski RT, Wactawski-Wende J et al (2012) Estrogen plus progestin and colorectal cancer incidence and mortality. J Am Soc Clin Oncol 30, 3983-3990

4. Ariazi EA, Brailoiu E, Yerrum S et al (2010) The G protein-coupled receptor GPR30 inhibits proliferation of estrogen receptor-positive breast cancer cells. Cancer Res 70, 1184-1194

5. Wang C, Lv X, He C et al (2013) The G protein-coupled estrogen receptor agonist G-1 suppresses proliferation of ovarian cancer cells by blocking tubulin polymerization. Cell Death Dis 4, e869
6. Girgert R, Emons G and Grundker C (2012) Inactivation of GPR30 reduces growth of triple-negative breast cancer cells: possible application in targeted therapy. Breast Cancer Res Treat 134, 199-205

7. Chan QKY, Lam HM, Ng CF et al (2010) Activation of GPR30 inhibits the growth of prostate cancer cells through sustained activation of Erk1/2, c-jun/c-fos-dependent upregulation of $\mathrm{p} 21$, and induction of $\mathrm{G} 2$ cell-cycle arrest. Cell Death Differ 17, 1511-1523

8. Bologa CG, Revankar CM, Young SM et al (2006) Virtual and biomolecular screening converge on a selective agonist for GPR30. Nature chemical biology 2, 207-212

9. Prossnitz ER and Barton M (2014) Estrogen biology: new insights into GPER function and clinical opportunities. Mol Cell Endocrionol 389, 71-83

10. Prossnitz ER and Barton M (2011) The G-protein-coupled estrogen receptor GPER in health and disease. Nat Rev Endocrinol 7, 715-726

11. Teng J, Wang ZY, Prossnitz ER, and Bjorling DE (2008) The GPER inhibits human urothelial cell proliferation. Endocrinology 149, 4024-4034

12. Cortes E, Sarper M, Robinson B et al (2018) GPER is a mechanoregulator of pancreatic stellate cells and the tumor microenvironment. EMBO Rep e46556

13. Sano R and Reed JC (2013) ER stress-induced cell death mechanisms. Biochim Biophys Acta 1833, 3460-3470

14. Tsai TC, Lai KH, and Su JH et al (2018) 7-Acetylsinumaximol B Induces Apoptosis and Autophagy in Human Gastric Carcinoma Cells through Mitochondria Dysfunction and Activation of the PERK/elF2 $\alpha /$ ATF4/CHOP Signaling Pathway. Mars Drugs 16, E104

15. Wang X, Xia Y, Xu CT et al (2017) ER membrane protein complex subunit 6 (EMC6) is a novel tumor suppressor in gastric cancer. BMB Rep 50, 411-416 